\title{
Revolution in der \\ ambulanten Pflege
}

\section{Daniel Lüthi}

Freier Journalist und Fotograf, Medientrainer, Bern

Selbst-Organisation statt von oben verordnete, unkoordinierte Aktivitäten, autonome Teams statt teure Manager, Menschlichkeit statt Bürokratie - und die Patienten im Mittelpunkt: Dies die Grundsätze der Non-Profit-Organisation Buurtzorg (Nachbarschaftshilfe), die seit 2006 in den Niederlanden ein revolutionäres neues Modell ambulanter Pflege praktiziert. Der charismatische Gründer Jos de Blok hat an einer Tagung in Olten mit seiner Erfolgsgeschichte die Fachleute berührt und beeindruckt.

Die Fachhochschule Nordwestschweiz und Spitex Bern hatten die Tagung gemeinsam organisiert - und damit einige der dort diskutierten Schlüsselbegriffe gleich in die Tat umgesetzt: Eigeninitiative, geteilte Verantwortung, Informationstransfer, Zusammenarbeit und Vernetzung. Was auf den ersten Blick komplex und kompliziert wirkt, ist es in Wirklichkeit nicht: «Es ist alles viel einfacher, als wir meinen", sagt Jos de Blok (siehe auch das Interview mit ihm auf der nächsten Seite). Unser Gesundheitssystem ist fragmentiert, die Hierarchien bringen nicht die gewünschten Resultate, die Strukturen kosten viel - zu viel. Alle sind unzufrieden, Patienten und Profis. Also ändern wir das System.

\section{Beeindruckendes Wachstum}

De Blok hat es getan, und die Entwicklung seiner Organisation ist einzigartig: Er startete 2007 mit einem Team, das vier Pflegende vereinte, einer davon war er selber. Heute umfasst Buurtzorg allein in den Niederlanden 800 weitgehend autonome Teams mit insgesamt etwa 9500 Pflegenden, diese betreuen rund 70000 Patientinnen und Patienten pro Jahr. Die meisten sind alte Menschen, viele davon leiden unter einer Demenz oder werden palliativ umsorgt in ihrem letzten Lebensabschnitt.

Im Hintergrund arbeiten 45 Personen, die den Teams die administrativen Arbeiten abnehmen, und 15 Coaches, die sie beraten und begleiten. "Manager haben wir keine», sagt Jos de Blok. Und damit auch keine unnötigen und kostspieligen Sitzungen, wie er sie in seinem früheren Berufsleben selber zahlreich erlebt hat.

\section{Gelebte Gemeinschaft}

Charakteristisch sind die geographische und die emotionale Nähe der Teams zu ihrem Klientel. Gelebte Gemeinschaft und horizontale Kommunikation sind zentral. Angehörige, Nachbarn und Freiwillige gehören zum Netzwerk. Das System basiert auf Vertrauen auch in die professionellen Kompetenzen, den guten Willen und das persönliche Engagement der Angestellten. «Empowerment» ist eine der Losungen. «Natürlich gibt es auch bei uns ab und zu ein faules Ei», antwortete Jos de Blok auf eine Frage aus dem Publikum. "Aber auch dies regelt das Team.»

\section{Elektronische Plattform}

Informationen fliessen weitgehend via «BuurtzorgWeb», eine eigene IT-Plattform, alle Angestellten sind mit einem Tablet ausgerüstet. Die Software hat die Organisation selber entwickelt, aufgrund der Bedürfnisse der Pflegenden und ihrer Patienten. "Herkömmliche Software dient vor allem der Finanz- und der IT-Abteilung eines Unternehmens», kommentierte Jos de Blok. Jedes Team verwaltet selbständig ein Budget von 350000 Euro pro Jahr, drei Prozent davon investieren die einzelnen Teams gemäss ihren Bedürfnissen und Interessen fix in Weiterbildungen. Zurzeit erzielt Buurtzorg einen Umsatz von etwa 300 Millionen Euro pro Jahr. Gemäss einer unabhängigen Untersuchung hat die Organisation die Kosten pro Patient fast halbiert, die Arbeitszufriedenheit gleichzeitig aber verdoppelt. Auch bei der Patientenzufriedenheit sind die Werte sehr hoch. Das bedeutet: Die Resultate der Qualitätskontrolle sind Teil dieser Erfolgsgeschichte. 


\section{«Es geht um Vertrauen»}

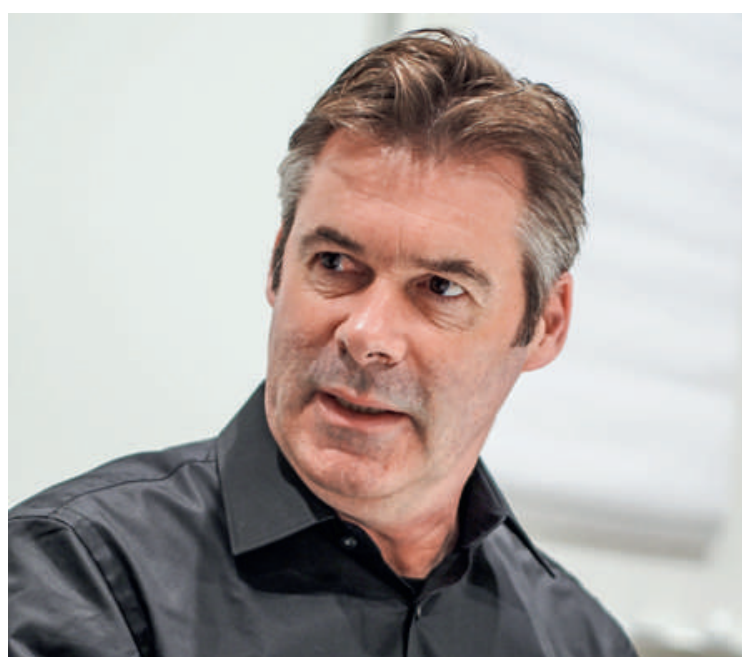

Mit 4 Personen hat Jos de Blok angefangen, heute arbeiten in seinen Teams allein in den Niederlanden 9500 Pflegende.

SÄZ: Ihre Organisation funktioniert mit Teams und Netzwerken. Was ist die Rolle der Ärztinnen und Ärzte darin?

Jos de Blok: Von Beginn weg haben wir Pflegende und Sozialarbeiter mit Allgemeinmedizinern und Hausärzten gut vernetzt. Wir haben diese wichtige Zusammenarbeit bewusst wiederbelebt, die in den siebziger und achziger Jahren gut funktioniert hatte und dann fast verloren ging, als die Aktivitäten im Gesundheitssektor immer mehr segmentiert und gesplittet wurden. Viele unserer Teams haben ihre Basis in Gesundheitszentren, da gibt es nebst Ärzten beispielsweise auch Physiotherapeutinnen.

\section{Sind Ärzte Mitglieder Ihrer Teams?}

Nein, nicht direkt. Je nach den Bedürfnissen der Patienten rufen wir Ärzte, auch Spezialisten, gezielt dazu. Ansonsten haben sie ihre eigene Routine, und die ist für unser System und unsere Arbeitsweise nicht dienlich. Wie auch immer: Unser Fokus liegt bei den Patienten, nicht bei den Teams.

Wie funktioniert nach Ihrer Erfahrung die «horizontale Kommunikation", von der Sie immer wieder sprechen, zwischen Medizinern und Pflegenden?

Es geht um Vertrauen. Wenn die Ärzte sehen, wie kompetent die Pflegenden sind, spielen Hierarchien und Standesdünkel keine Rolle mehr. Da hat auch ein Wan- del stattgefunden: Viele Ärzte haben gemerkt, dass Entscheide besser im Team gefällt werden und sie auch mit den Patienten sprechen sollten. Im Übrigen gibt es ja in der Ärzteschaft immer mehr Frauen, und diese sind in der Regel mehr auf Zusammenarbeit fokussiert, haben weniger Status-Probleme als Männer.

\section{Stimmt es, dass Sie Ihr Modell künftig nicht nur im ambulanten, sondern auch im stationären Bereich praktizieren wollen?}

Ja, wir wollen auch hier Alternativen schaffen und sind daran, Nachbarschafts-Kliniken zu schaffen. Demente Menschen zum Beispiel sollten möglichst lange in ihrer vertrauten Umgebung und Gemeinschaft bleiben können.

\section{Die Erfolgsgeschichte Buurtzorg gibt es inzwischen ja auch ausserhalb der Niederlande, in England, Schweden, den USA, Japan und China beispielsweise. Ist sie nach Ihrer Einschätzung auch in der Schweiz möglich?}

Überall läuft der Prozess ein wenig anders. Wichtig ist, dass möglichst viele verschiedene Beteiligte unser Modell als Fortschritt sehen: Pflegende, Ärzte, Politiker und so weiter. In der Schweiz möchten viele Pflegefachleute sicher so arbeiten wie wir, aber die Versicherungen sind zu dominant. Sie sagen, was und wie viel ein $\mathrm{Pa}$ tient zugute hat. Unsere Teams arbeiten weitgehend autonom, sie entwickeln Lösungen gemeinsam mit den Patienten und ihren Angehörigen. Was die Finanzen betrifft, denken wir nicht in Aktivitäten pro Zeiteinheit, sondern in Kosten pro Klient. Und da fallen bei uns unter dem Strich weniger Stunden an als in einem herkömmlichen System, wie es beispielsweise in der Schweiz praktiziert wird.

\section{Was bräuchte es, damit Sie auch hierzulande aktiv würden?}

Die Initiative muss aus dem jeweiligen Land kommen Beispielsweise von Leuten, die ein Team gebildet haben oder bilden wollen, und nach unseren Grundsätzen arbeiten möchten. Ich würde sie unterstützen. Vorderhand rege ich Diskussionen an, auch auf politischer Ebene. Es ist wie bei einem Patienten: Solange er sein eigenes Problem nicht sieht und nicht versteht, passiert nichts ... 